\title{
3D SELF-ASSEMBLED MICROSCALE RESONATOR AS ULTRA SENSITIVE ISOTROPIC SENSOR
}

\author{
Kriti Agarwal, Chao Liu, Daeha Joung, and Jeong-Hyun Cho
}

Department of Electrical and Computer Engineering, University of Minnesota, Minneapolis, Minnesota, USA

\section{ABSTRACT}

Sensors based on split-ring resonators have long been explored as viable candidates for biological applications since their frequency is a function of the permittivity of surrounding media; plagued by their detection ability being confined to the gap, they demonstrate a low sensitivity. In addition, the high directionality of their response has been a major limiting factor in the advancement of these resonant structures, which renders the spectrum incomprehensible. Thus, this study demonstrates the use of origami like self-folding to achieve a three-dimensionally coupled novel split-ring resonator which is invariant under any rotation and has ultra-high sensitivity.

\section{INTRODUCTION}

Pendry's design of a split-ring resonator array presented a technique for the realization of left-handed metamaterial [1]. The resonant frequency of these resonators is a function of their length, split size, angle of incidence of light $(\theta)$ (Fig. 1), and the permittivity of the surroundings. The resonance dependence on the permittivity of the surroundings allows their use as biological sensors which can predict changes in the cell life cycle due to changes in cell permittivity as a result of chemical reactions within the cell membrane [2]. However, the detection ability of the splitring resonator is limited only to the split in the resonant structure, which is much smaller than the total length of the resonator [3]. Furthermore, for their efficient function as a sensor it is necessary that any changes in the transmission spectrum be solely dependent on the changes in permittivity. But, the $\mathrm{C}$-shape had a major disadvantage due to the high dimensionality of its response; as the resonator was rotated, the resonance shifted from magnetic to electrical (Fig. 1), creating an ambiguity in the signal transduced. If the same resonator is now forced to couple isotropically in a three-dimensional (3D) configuration, then each resonator experiences the same exact surroundings as its neighboring resonators; causing the entire structure to demonstrate a very strong resonance at a single frequency. Changes in the resonant frequency of any one of the resonators has a domino effect causing amplification in the transduced signal.

\section{ANALYSIS AND RESULTS}

The 3D folded structure with 3D star resonators (Fig. 2a, b), demonstrated a single fundamental resonant frequency. At the resonant frequency, the cubic structure did not show any changes in transmission on rotating along any/all three axis (fig 2c), as opposed to its 2D counterpart which had a highly dimensional response. The surface current of the cubic structure at the resonant frequency demonstrated a smooth and equal surface current across all the resonators, proving that it is the 3D coupling that is inducing the isotropic response in the cubic structure.

The 3D coupling of the resonator, not only increases the isotropy of the split-ring resonator, it also magnifies its sensitivity. Since each resonator induces a resonance in its neighboring resonators, a local permittivity change experienced by any one of the resonators has a ripple effect that enhances the transduced change in the transmission spectrum. The isotropic 3D star resonator thus has an ultra-high sensitivity which is 10 times higher than its two dimensional counterpart (Fig 2d). Through the use of an origami self-folding technique [4], the resonators patterned on a $20 \mu \mathrm{m}$ thick substrate could be folded into a $200 \mu \mathrm{m}$ sized cubic structure through the use of surface tension force caused by the reflow of a polymer hinge on heating (Fig $2 b$ ).

The 3D star shaped split-ring resonator patterned on the face of $3 \mathrm{D}$ cubic substrate demonstrates an isotropic ultra-high sensitivity resonant structure. The isotropy of the response and the ripple effect on resonance of the structure make it an excellent candidate for use in high sensitivity chemical and biological applications.

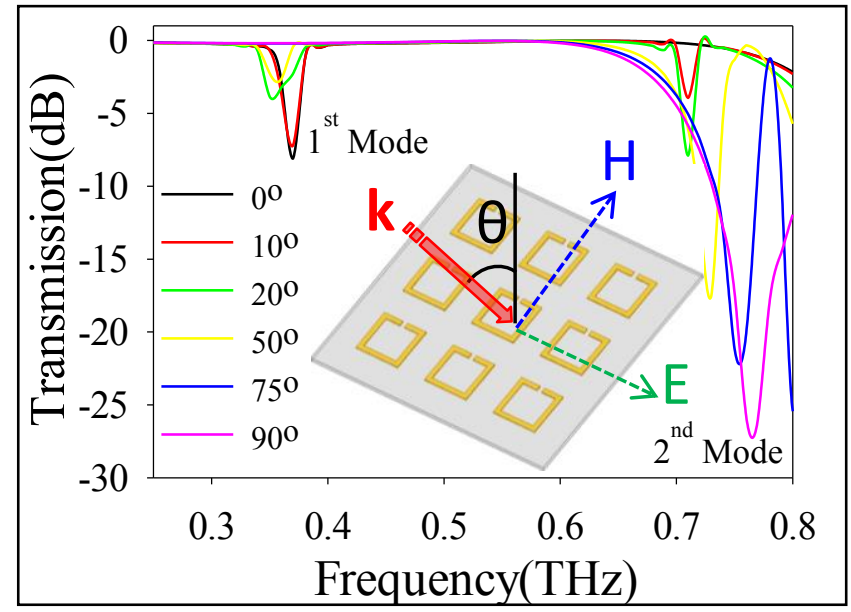

Figure 1: Simulated transmission characteristics of a $2 D C$-shaped resonator. Demonstrating the directional transmission response shifting from magnetic (1st mode) to electrical resonance (2nd mode) as a result of varying angle of incidence $(\theta)$ from $0^{\circ}$ to $90^{\circ}$.

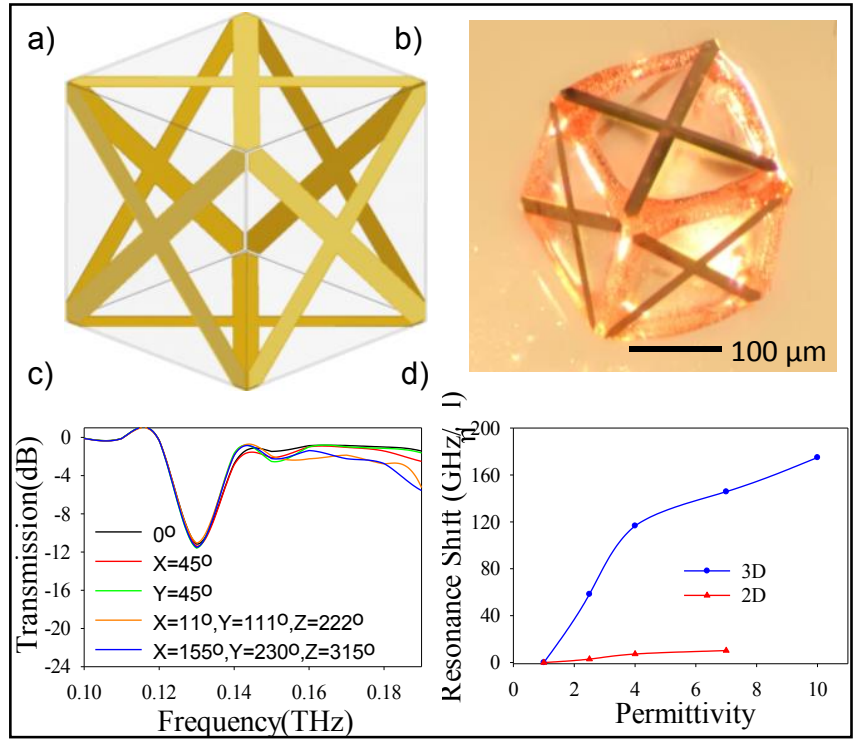

Figure 2: (a) Illustration of isotropic 3D star resonator (b) Optical images of the $3 D$ resonator. (c) Simulated transmission response of the cube showing an isotropic behavior, (d) Resonant frequency changes of $2 D$ and $3 D$ resonators. 


\section{ACKNOWLEDGEMENT}

This material is based upon work supported by a start-up fund at the University of Minnesota, Twin Cities and an NSF CAREER Award (CMMI-1454293). Travel support has been generously provided by the Transducer Research Foundation.

\section{REFERENCES}

[1] Pendry, John B., et al. "Magnetism from conductors and enhanced nonlinear phenomena." Microwave Theory and
Techniques, IEEE Transactions on 47.11 (1999), 2075-2084.

[2] Saito, Hiroshi, et al. "Measurement of complex permittivity for biological cells at $1.7-2.6 \mathrm{GHz}$ by waveguide penetration method." Proc. URSI XXIX General Assembly (2008).

[3] Park, S. J., et al. "Detection of microorganisms using terahertz metamaterials." Scientific reports 4 (2014).

[4] Cho, Jeong-Hyun, et al. "Self-assembly of lithographically patterned nanoparticles." Nano letters 9.12 (2009), 40494052. 